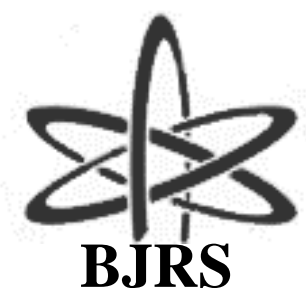

\author{
BRAZILIAN JOURNAL \\ $\mathrm{OF}$ \\ RADIATION SCIENCES \\ 06-02-B (2018) 01-17
}

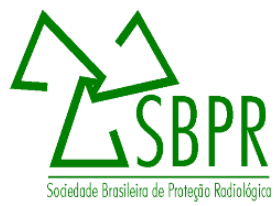

\title{
Desenvolvimento e avaliação de técnica de monitoração in vivo de radionuclídeos de alta energia nos pulmões em situações de emergência
}

\author{
Mello $^{a}$ J. Q., Lucena ${ }^{a}$ E. A., Dantas ${ }^{a}$ A.L.A, Dantas B.M. \\ ${ }^{a}$ Instituto de Radioproteção e Dosimetria, 22783-127, Av. Salvador Allende s/n-Barra da Tijuca, \\ Rio de janeiro-RJ, Brasil \\ jaquelinequince@hotmail.com; bmdantas@ird.gov.br
}

\section{RESUMO}

Situações de emergência em centrais nucleares envolvendo liberação de materiais radioativos requerem a utilização de técnicas de monitoração de indivíduos expostos a riscos de incorporação de radionuclídeos. Tais técnicas devem fornecer resultados rapidamente e apresentar sensibilidade suficiente para a deteção de atividades que resultem em doses internas compatíveis com os limites de exposição. Uma grande variedade de radionuclídeos pode ser identificada e quantificada por meio de técnicas de monitoração in vivo no corpo humano e sua disponibilidade é de grande importância nas ações de remediação. Este trabalho apresenta o desenvolvimento e validação de métodos de monitoração in vivo para avaliação da incorporação de radionuclídeos via inalação. Os sistema de monitoração compostos por detetores $\mathrm{NaI}(\mathrm{Tl}) 8 \times 4$ e NaI(Tl)3x3 ambos instalados no Contador de Corpo Inteiro e $\mathrm{NaI}(\mathrm{TI}) 3 \times 3$ instalado na saleta de alta atividade do IRD foram calibrados com um simulador de tórax e pulmão tipo LLNL contendo ${ }^{152}$ Eu. Foram calculadas as eficiências de deteção correspondentes às energias dos fótons mais intensos do ${ }^{152}$ Eu e obtida uma curva de calibração na faixa de 100 a $1400 \mathrm{keV}$. A partir desta curva foram calculadas as eficiências de deteção, as atividades mínimas detetáveis e as doses efetivas mínimas detetáveis correspondentes aos radionuclídeos de maior interesse no caso de um acidente com liberação de pluma radioativa, como por exemplo, ${ }^{134} \mathrm{Cs},{ }^{137} \mathrm{Cs}$ e ${ }^{106} \mathrm{Ru}$. As técnicas apresentam sensibilidade adequada para monitoração in vivo em tais situações, sendo capaz de detetar atividades que resultem em doses efetivas comprometidas na ordem de microsieverts, para os cenários de exposição apresentados.

Palavras-chave: Radionuclídeos, Dosimetria Interna e Radioproteção. 


\begin{abstract}
Emergency situations in nuclear power plants involving the release of radioactive materials require the use of monitoring techniques for individuals exposed to risks of radionuclide incorporation. Such techniques should provide rapid results and provide sufficient sensitivity for the detection of activities that result in internal doses consistent with exposure limits. A wide variety of radionuclides can be identified and quantified by in vivo monitoring techniques in the human body and their availability is of great importance in remediation actions. This work presents the development and validation of in vivo monitoring methods for the evaluation of inhaled radionuclide incorporation. The monitoring systems composed of $\mathrm{NaI}$ (Tl) $8 \times 4$ and $\mathrm{NaI}$ (Tl) $3 \times 3$ detectors both installed in the Whole Body Counter and NaI (Tl) 3x3 installed in the high activity room of the IRD were calibrated with a chest and lung simulator type LLNL containing ${ }^{152} \mathrm{Eu}$. The detection efficiencies corresponding to the most intense photon energies of ${ }^{152} \mathrm{Eu}$ were calculated and a calibration curve in the range of 100 to 1400 $\mathrm{keV}$ was obtained. From this curve the detection efficiencies, the minimum detectable activities and the detectable minimum effective doses corresponding to radionuclides of major interest in the case of a radioactive plume-releasing accident, such as, for example, ${ }^{134} \mathrm{Cs},{ }^{137} \mathrm{Cs}$ and ${ }^{106} \mathrm{Ru}$ were calculated. The techniques present adequate sensitivity for in vivo monitoring in such situations, being able to detect activities that result in effective doses committed in the order of microsieverts, for the exposure scenarios presented.
\end{abstract}

Keywords: Radionuclides, Internal Dosimetry and Radioprotection.

\title{
INTRODUÇÃO
}

Situações de emergência envolvendo riscos de incorporação de materiais radioativos requerem a atuação de equipes capacitadas a realizar monitoração interna de radionuclídeos, tanto em trabalhadores quanto em indivíduos do público.

A aplicação de metodologias para monitoração da exposição interna deve considerar a sensibilidade dos sistemas de deteção para identificação e quantificação dos radionuclídeos críticos em relação à sua possível ocorrência em situações de acidentes radiológicos e nucleares. [1]

Acidentes envolvendo liberação de aerossóis na forma de particulados ou gases contendo materiais radioativos representam um risco de exposição interna, via inalação, nos locais próximos às áreas afetadas. Em consequência dos acidentes nucleares de Chernobyl e Fukushima, radionuclídeos emissores gama de alta energia, tais como ${ }^{85} \mathrm{Kr},{ }^{95} \mathrm{Zr},{ }^{95} \mathrm{Nb},{ }^{106} \mathrm{Ru},{ }^{127} \mathrm{Sb},{ }^{131} \mathrm{I},{ }^{132} \mathrm{I}$, 
${ }^{132} \mathrm{Te},{ }^{134} \mathrm{Cs},{ }^{136} \mathrm{Cs},{ }^{137} \mathrm{Cs},{ }^{140} \mathrm{Ba},{ }^{144} \mathrm{Ce},{ }^{239} \mathrm{~Np}$, entre outros, foram considerados críticos, do ponto de vista de exposição interna do público e dos trabalhadores. [2]

\section{MATERIAIS E MÉTODOS}

O presente estudo foi desenvolvido nas instalações do Laboratório de Monitoração in vivo (LABMIV) do Instituto de Radioproteção e Dosimetria IRD-CNEN, utilizando detetores $\mathrm{NaI}(\mathrm{Tl}) 8 \times 4$ e $\mathrm{NaI}(\mathrm{Tl}) 3 \times 3$ que se encontram instalados na Unidade de Contador de Corpo Inteiro (UCCI) e o detetor $\mathrm{NaI}(\mathrm{Tl}) 3 \times 3$ instalado na saleta de alta atividade. A sala UCCI é composta de paredes de aço de $15 \mathrm{~cm}$ de espessura e dimensões internas de 2,50m x 2,50m x 2,62m, revestidas internamente com camadas adicionais de chumbo, cádmio e cobre com espessuras de $3 \mathrm{~mm}, 1,5 \mathrm{~mm}$ e $0,5 \mathrm{~mm}$ respectivamente [4]. A saleta de alta atividade é composta por três paredes de alvenaria revestidas à meia altura com uma camada de chumbo com espessura de 1,0 mm, e chão de alvenaria revestido de chumbo com espessura de 5,0 cm [5].

Para as geometrias de contagens foi utilizado um simulador de Tórax tipo LLNL e um par de pulmões de resina de poliuretano expandido produzidos pela Universidade de Cincinatti, contendo um total de $31875 \pm 385 \mathrm{~Bq}$ de ${ }^{152} \mathrm{Eu}$.

\subsection{Calibração do sistema de deteção}

A metodologia de calibração consiste na obtenção de curvas "Eficiência x Energia", utilizando as energias dos fótons de radiação gama mais intensos emitidos pelo ${ }^{152} \mathrm{Eu}$, a saber: 121,8; 344,3; 778,$9 ; 1112,1$ e $1408,0 \mathrm{keV}$. Uma das dificuldades encontradas quando o ${ }^{152} \mathrm{Eu}$ é utilizado para calibração de detetores de iodeto de sódio reside na baixa resolução em energia deste cristal cintilador além da possível ocorrência de contribuição do efeito soma, quando a distância fonte detector é menor que $10 \mathrm{~cm}$. Nas condições de medições de baixa atividade em que as amostras precisam estar próximas ao detector, é recomendável que seja feita a correção deste fenômeno [6]. Quando for imprescindível o uso do ${ }^{152} \mathrm{Eu}$, deverão ser programados estudos onde a distância fonte detector possa ser variada e, assim, corrigida esta contribuição. Este trabalho não levou este fenômeno em consideração, pois se trata de uma técnica de avaliação preliminar em campo, visando 
uma primeira resposta a situações de emergência. Em caso de resultados positivos, medições mais precisas seriam realizada em um contador de corpo inteiro, em que tais fenômenos radiométricos seriam levados em consideração, ou, alternativamente, a geometria de deteção seria otimizada de forma a minimizá-los.

Para cada geometria avaliada, foram realizadas cinco medições de 2700 segundos, e obtidos os valores de contagens para cada região de interesse (RDI), com o detetor posicionado à distância de $2 \mathrm{~cm}$, coincidente com as marcações existentes na parte frontal do simulador: centro do tórax, pulmão direito e pulmão esquerdo. Cada sistema de deteção foi avaliado nas três geometrias de posicionamento do detetor em relação ao tórax.

O Sistema de deteção \#1 é composto por um detetor cintilador tipo $\mathrm{NaI}(\mathrm{TI}) 3 \times 3$ instalado na saleta de alta atividade. O simulador é posicionado na vertical, em geometria de indivíduo monitorado em posição sentada, como mostra figura 1.

Figura 1. Sistema \#1: Detetor NaI(TI) $3 \times 3$ instalado na saleta de alta atividade posicionado no centro do simulador, no pulmão direito e no pulmão esquerdo respectivamente.
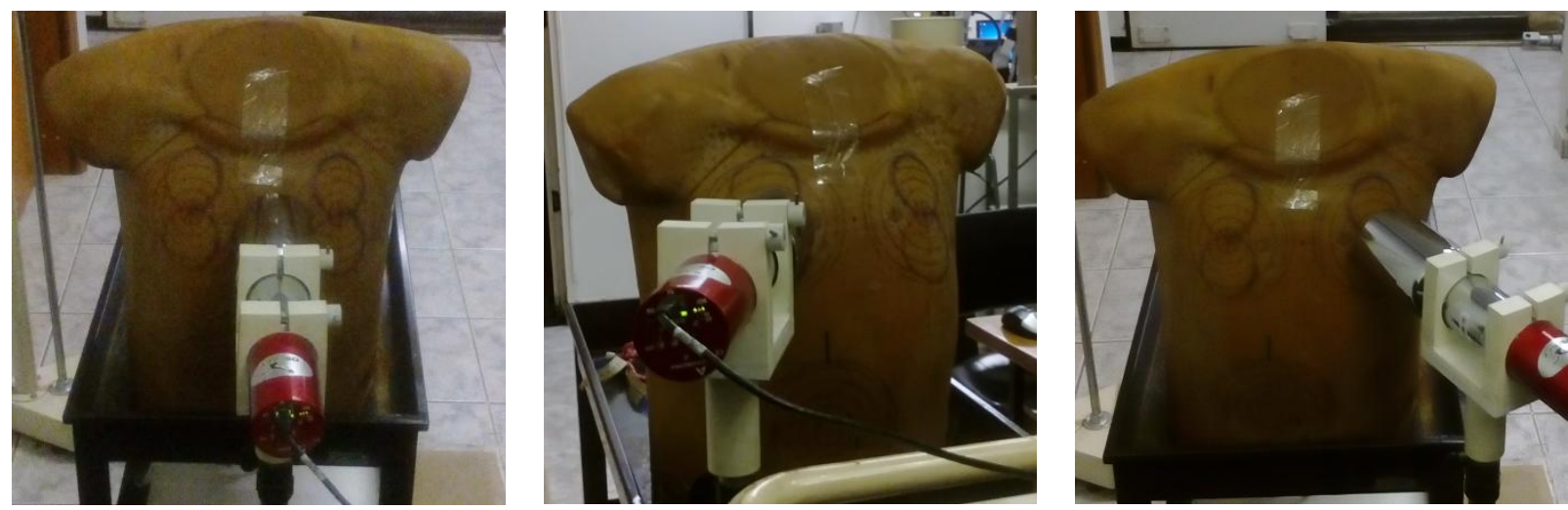

O sistema de deteção \#2 é composto por um detetor cintilador tipo NaI(TI)3x3 colimado com chumbo, instalado na unidade do contador de corpo inteiro UCCI. O simulador é apoiado na cadeira com inclinação fixa, em geometria de monitoração de indivíduo reclinado, como mostra a figura 2. 
Figura 2. Sistema \#2: Detetor NaI(TI) $3 \times 3$ posicionado no centro do simulador, no pulmão direito e no pulmão esquerdo respectivamente.
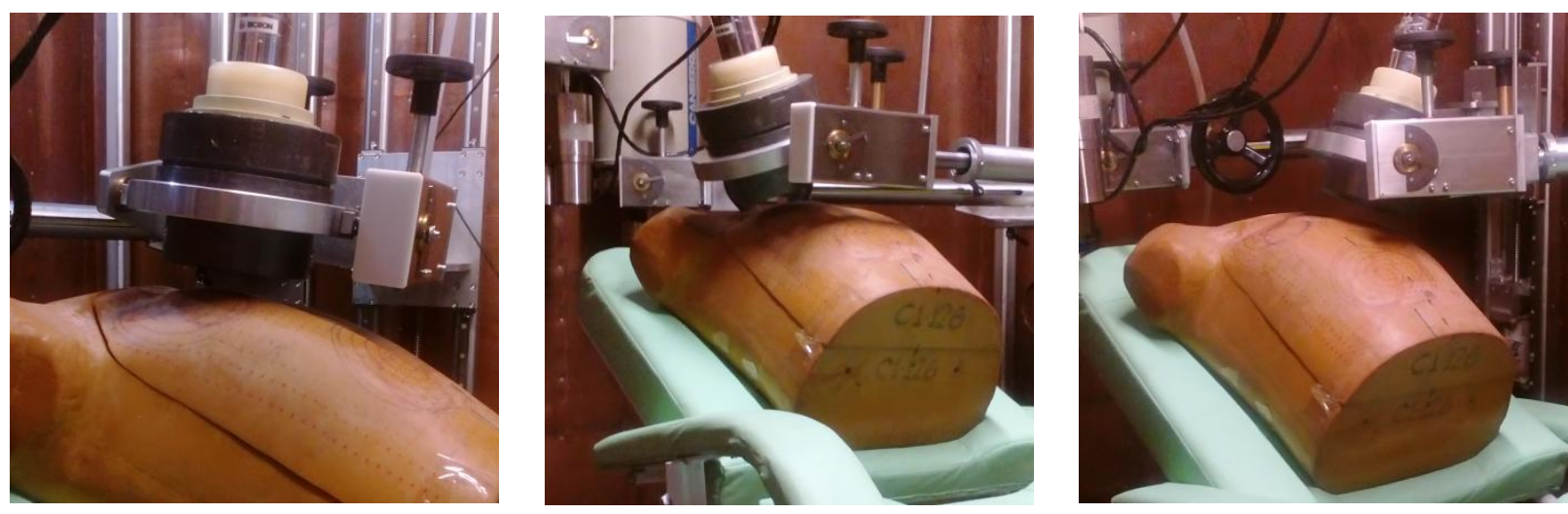

O sistema de deteção \#3 é composto por um detetor cintilador tipo NaI(TI) 8x4, instalado na sala blindada do Contador de Corpo Inteiro. O simulador é posicionado da mesma forma como no sistema \#2, como mostra a figura 3.

Figura 3. Sistema \#3: Detetor NaI(TI) $8 \times 4$ posicionado no centro do simulador, no pulmão direito e no pulmão esquerdo respectivamente.
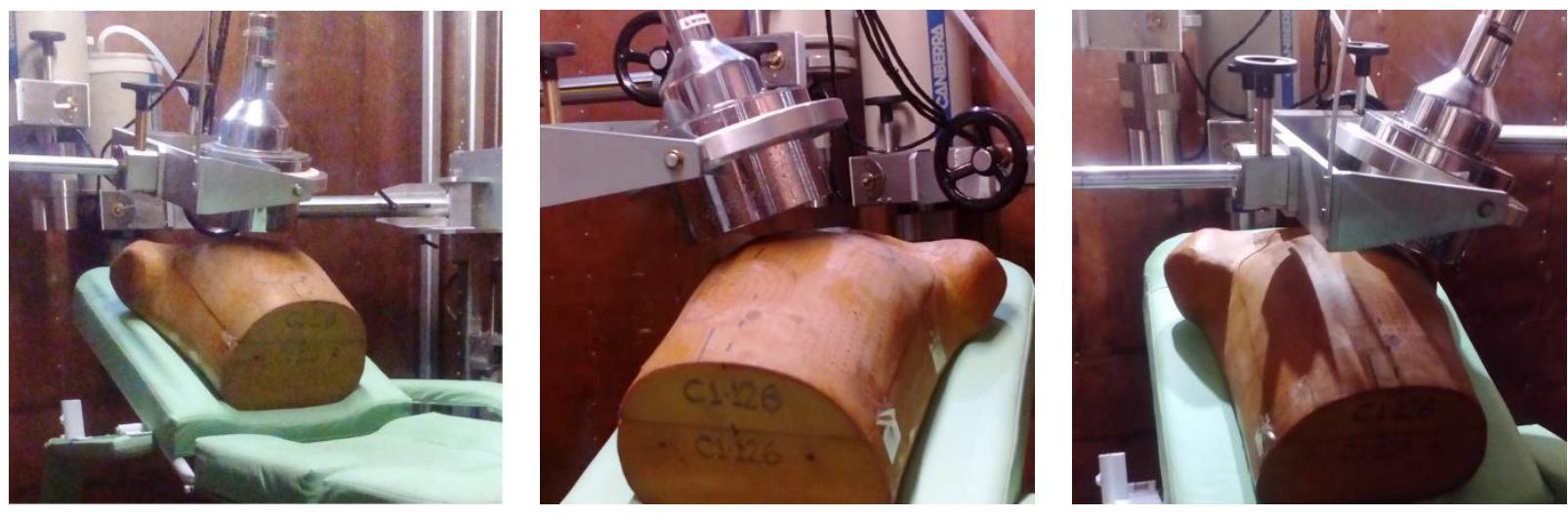

Em seguida foram realizadas medições de radiação de fundo (background) para cada sistema de deteção em um indivíduo não ocupacionalmente exposto. A figura 4 ilustra a medição em uma das geometrias para o sistema de deteção \#1. 
Figura 4. Medição do indivíduo não exposto utilizando o NaI(TI) $3 \times 3$ instalado na saleta da alta atividade.

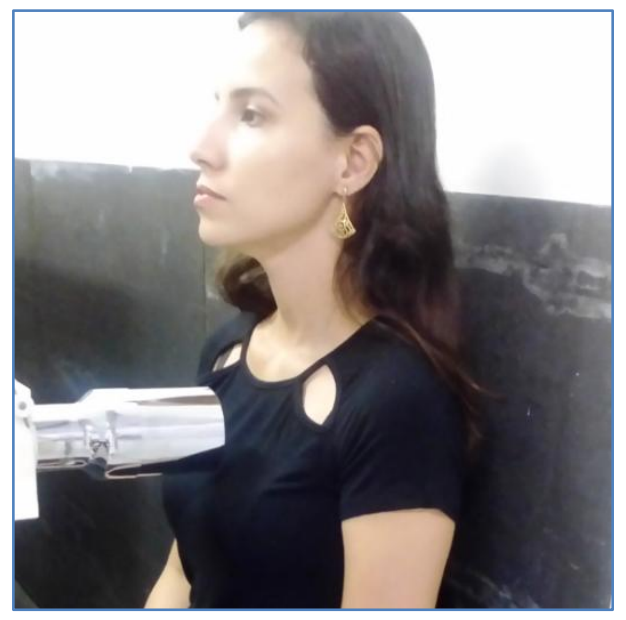

\subsection{Avaliação da sensibilidade da técnica}

No presente estudo, foram selecionados os radionuclídeos ${ }^{134} \mathrm{Cs}$, ${ }^{137} \mathrm{Cs}$ e ${ }^{106} \mathrm{Ru}$ para a avaliação da sensibilidade da técnica na monitoração in vivo nos pulmões. Estes radionuclídeos seriam liberados no caso de um acidente nuclear e apresentam coeficiente de dose interna relevante. Os três apresentam características de emissão (energia dos fótons e respectivas intensidades gama) que permitem a utilização de técnicas de monitoração in vivo. Além disso, possuem meia-vida física significativa, permanecendo presentes no ambiente por longos períodos após o acidente sendo, portanto, radionuclídeos críticos do ponto de vista da exposição de populações e trabalhadores envolvidos nas tarefas de remediação.

A razão entre a taxa de contagem (cps) e o produto da atividade (Bq) contida no simulador, corrigida para a data da calibração e a intensidade gama do fóton, expressa a eficiência de deteção que é calculada conforme a equação (1):

$$
E f(c p s / B q)=c p s / A t i v \cdot I g
$$

onde Ef é a eficiência de deteção; cps é a taxa de contagem do simulador na região de interesse; Ativ é a atividade em Bq e Ig é a intensidade gama do fóton emitido pelo radionuclídeo. 
Com base nos valores de eficiência para cada energia, é obtida a curva de calibração para a faixa de energia de 100 a $1400 \mathrm{keV}$.

Os parâmetros utilizados para a avaliação da sensibilidade correspondem à AMD (atividade mínima detetável), IMD (incorporação mínima detetável) e DEMD (dose efetiva mínima detetável).

A partir dos valores das eficiências de detecção, são calculados os respectivos valores de Atividade Mínima Detetável, Incorporação Mínima Detetável e Dose Efetiva Mínima Detetável, correspondentes aos três radionuclídeos selecionados ${ }^{134} \mathrm{Cs},{ }^{137} \mathrm{Cs}$ e ${ }^{106} \mathrm{Ru}$.

A AMD é calculada de acordo com a equação (2):

$$
A M D(B q)=4,65 \times \operatorname{Raiz}(N) / E f x T \times I g
$$

onde AMD é a atividade mínima detectável $(\mathrm{Bq})$ do radionuclídeo de interesse; $\mathrm{N}$ é o total de contagens do indivíduo não exposto na região de interesse; Ef é a eficiência de deteção; T é o tempo de contagem (segundos) e; Ig é a intensidade gama do fóton.

A Incorporação Mínima Detetável (IMD) é uma função direta da AMD e depende do cenário de exposição e do tempo decorrido entre a incorporação e a medição. A IMD é calculada de acordo com a equação (3):

$$
I M D=A M D / m(t)_{i n h}
$$

O último parâmetro a ser calculado é a Dose Efetiva Mínima Detetável (DEMD), que é diretamente proporcional ao valor da IMD e ao coeficiente de dose, e(g), específico para cada radionuclídeo de interesse, considerando o cenário de exposição adotado. A DEMD é calculada de acordo com a equação (4):

$$
D E M D=I M D i n h \times e(g)_{i n h}
$$

Os valores de "m(t)", fração de retenção no corpo humano $(\mathrm{Bq} / \mathrm{Bq})$ e "e(g)", coeficiente de conversão de dose $(\mathrm{mSv} / \mathrm{Bq})$, utilizados para os cálculos de IMD e DEMD são parâmetros biocinéticos e dosimétricos fornecidos pelo software AIDE [3]. Neste trabalho utiliza-se valores de $\mathrm{m}(\mathrm{t})$ correspondentes a 1 e 7 dias após a incorporação via inalação. Os valores de $\mathrm{m}(\mathrm{t})$ e e(g) estão representados na tabela 1. 
Tabela 1: Parâmetros biocinéticos e dosimétricos do ${ }^{134} \mathrm{Cs},{ }^{137} \mathrm{Cs}$ e ${ }^{106} \mathrm{Ru}$ utilizados para os cálculos de incorporação mínima detetável e dose efetiva mínima detetável, correspondentes ao cenário de incorporação de radionuclídeo via inalação.

\begin{tabular}{cccc}
\hline & $\mathbf{m}_{\mathbf{t}}($ inalação)/ AMAD 1 - composto M & \\
\cline { 2 - 3 } Radionuclídeo & $\mathbf{1}$ dia $(\mathbf{B q} / \mathbf{B q})$ & $\mathbf{7} \mathbf{d i a s}(\mathbf{B q} / \mathbf{B q})$ & \multirow{2}{*}{$\mathbf{e}(\mathbf{g})(\mathbf{m S v} / \mathbf{B q})$} \\
\hline${ }^{\mathbf{1 3 4}} \mathbf{C s}$ & $1,69 \times 10^{-1}$ & $9,85 \times 10^{-2}$ & $8,23 \times 10^{-6}$ \\
${ }^{\mathbf{1 3 7}} \mathbf{C s}$ & $1,70 \times 10^{-1}$ & $9,91 \times 10^{-2}$ & $8,84 \times 10^{-6}$ \\
${ }^{\mathbf{1 0 6}} \mathbf{R u}$ & $1,69 \times 10^{-1}$ & $9,78 \times 10^{-2}$ & $2,61 \times 10^{-5}$ \\
\hline
\end{tabular}

\subsection{Validação da técnica}

Com o objetivo de se validar a técnica apresentada, foram realizadas, na geometria central, medições do simulador de tórax contendo um par de pulmões de ${ }^{60} \mathrm{Co}$ com atividade padrão de $22510 \pm 223 \mathrm{~Bq}$. O procedimento foi realizado para os três sistemas de deteção avaliados. A partir de duas regiões de interesse de emissão gama do ${ }^{60} \mathrm{Co}$, foram obtidas contagens líquidas no tempo de 5 minutos e, utilizando-se a curva de eficiência de cada sistema detecção, foram calculadas as atividades, sendo as mesmas comparadas à atividade certificada do simulador padrão. A tabela 2 apresenta as regiões de interesse dos fótons gama emitidos pelo ${ }^{60} \mathrm{Co}$, utilizadas no presente trabalho.

Tabela 2: Energias correspondentes às emissões gama do ${ }^{60} \mathrm{Co}$ e suas respectivas regiões de interesse.

\begin{tabular}{lcc}
\hline & RDI 1 & RDI 2 \\
\hline Foto Pico (keV) & 1173 & 1332 \\
RDI (Canais) & $347-408$ & $414-470$ \\
RDI Energia (keV) & $1094-1250$ & $1265-1407$ \\
\hline
\end{tabular}




\section{RESULTADOS E DISCUSSÃO}

A tabela 3 apresenta as equações das curvas de eficiência de deteção para os três os sistemas avaliados. As tabelas 4 e 5 apresentam, respectivamente, os valores de eficiências obtidas por meio das curvas, bem como as AMDs, IMDs, DEMDs para os Sistemas de deteção \#1, \#2 e \#3, relativos à monitoração in vivo de ${ }^{134} \mathrm{Cs},{ }^{137} \mathrm{Cs}$ e ${ }^{106} \mathrm{Ru}$ nos pulmões.

Para a avaliação de incorporação e dose, foi considerado um cenário de inalação, supondo classe de solubilidade pulmonar da partícula como M (moderada) e tamanho aerodinâmico médio em atividade (AMAD) correspondente a 1 micrometro. Os valores calculador são referentes a 1 e 7 dias após a incorporação.

Tabela 3. Curvas de eficiência para os sistemas de deteção \# 1, 2 e 3 nas geometrias Central, Direita e Esquerda

\begin{tabular}{|c|c|c|c|}
\hline \multicolumn{2}{|c|}{ Geometria } & \multirow{2}{*}{$\begin{array}{c}\text { Curva Ef. } x \text { En. (cps/dps) } \\
y=\mathbf{0 , 0 0 6 7} \mathrm{e}^{-0,0008 x}\end{array}$} & \multirow{2}{*}{$\frac{\mathbf{R}^{2}}{0,9558}$} \\
\hline Sistema \#1 & Central & & \\
\hline $\mathrm{NaI}(\mathrm{Tl}) 3 \times 3$ & Direito & $y=0,0078 e^{-0,0008 x}$ & 0,9562 \\
\hline Saleta & Esquerdo & $y=0,0046 e^{-0,0006 x}$ & 0,8885 \\
\hline Sistema \#2 & Central & $y=\mathbf{0 , 0 0 2 0} e^{-0,0007 x}$ & 0,7724 \\
\hline $\mathrm{NaI}(\mathrm{Tl}) 3 \times 3$ & Direito & $y=0,0028 e^{-0,0009 x}$ & 0,9190 \\
\hline $\mathrm{CCI}$ & Esquerdo & $y=0,0019 e^{-0,0008 x}$ & 0,8911 \\
\hline Sistema \#3 & Central & $\mathrm{y}=\mathbf{0 , 0 2 5 1} \mathrm{e}^{-0,0005 \mathrm{x}}$ & 0,9488 \\
\hline $\mathrm{NaI}(\mathrm{Tl}) 8 \mathrm{x} 4$ & Direito & $y=0,0237 e^{-0,0005 x}$ & 0,8979 \\
\hline $\mathrm{CCI}$ & Esquerdo & $y=0,0208 e^{-0,0006 x}$ & 0,8581 \\
\hline
\end{tabular}

Tomando-se como base de comparação a geometria Central, observa-se que o sistema \#3 (NaI 8x4 instalado na sala blindada do Contador de Corpo Inteiro) apresenta valor de eficiência inicial $(\mathbf{0 , 0 2 5 1} \mathbf{~ c p s} / \mathbf{d p s}$, para $\mathrm{En}=0 \mathrm{keV})$ cerca de 10 vezes superior ao valor $(\mathbf{0 , 0 0 2 0} \mathbf{~ c p s} / \mathbf{d p s})$ obtido no 
sistema \#2 (NaI 3x3 na UCCI), e 4 vezes superior ao valor ( $\mathbf{0 , 0 0 6 7}$ cps/dps) obtido com o sistema \#1 (NaI 3x3 na saleta de alta atividade). Estas diferenças de eficiência de deteção podem ser atribuídas ao maior volume do cristal de NaI do sistema \#3.

A diferença observada entre os dois sistemas que utilizam detetores NaI 3x3 (Sistema \#1 e \#2) se justifica pelo fato do sistema \#2 ser instalado no interior do colimador de $\mathrm{Pb}$, a $5 \mathrm{~cm}$ de profundidade, de tal forma que a distância entre a superfície do simulador e a face frontal do cristal do sistema \#2 é maior do que no sistema \#1, que não possui colimador.

A geometria de pulmão direito apresenta maior sensibilidade para o sistema $\mathrm{NaI}(\mathrm{Tl}) 3 \times 3 \mathrm{na}$ saleta, como mostra a tabela 4. Isto se deve ao fato do pulmão esquerdo apresentar menor volume, pois partilha o espaço torácico com o coração. Assim, a maior deposição de material radioativo ocorre no pulmão direito, apresentando este maior atividade e, consequentemente, maior contribuição nas contagens para esta geometria.

Tabela 4. Sensibilidade da técnica para monitoração in vivo de ${ }^{134} \mathrm{Cs}$, ${ }^{137} \mathrm{Cs}$ e ${ }^{106} \mathrm{Ru}$ nos pulmões considerando incorporação de partículas tipo M e AMAD 1 micrômetro. Calibração realizada na saleta com o detetor $\mathrm{NaI}(\mathrm{TI}) 3 \times 3$ (Sistema \#1), nas posições central, direito e esquerdo do simulador.

\begin{tabular}{|c|c|c|c|c|c|c|c|}
\hline Geometria & Radionuclídeo & $\begin{array}{c}\text { Ef } \\
(\mathbf{c p s} / \mathbf{B q})\end{array}$ & $\begin{array}{l}\operatorname{AMD}(\mathrm{Bq}) \\
(\mathrm{T}=300 \mathrm{~s})\end{array}$ & $\begin{array}{l}\text { IMD } \\
(\mathrm{Bq}) \\
1 \text { dia }\end{array}$ & $\begin{array}{c}\text { IMD } \\
(\mathrm{Bq}) \\
7 \text { dias }\end{array}$ & $\begin{array}{c}\text { DEMD } \\
1 \text { dia } \\
\left(\mathbf{x 1 0}^{-2}\right) \\
(\mathrm{mSv})\end{array}$ & $\begin{array}{c}\text { DEMD } \\
7 \text { dias } \\
\left(\mathrm{x}^{-2}\right) \\
(\mathrm{mSv})\end{array}$ \\
\hline \multirow{3}{*}{ Central } & ${ }^{134} \mathrm{Cs}$ & 0,0035 & 322 & 1905 & 3268 & 1,6 & 2,7 \\
\hline & ${ }^{137} \mathrm{Cs}$ & 0,0039 & 347 & 2039 & 3498 & 1,8 & 3,1 \\
\hline & ${ }^{106} \mathrm{Ru}$ & 0,0044 & 484 & 2862 & 4946 & 7,5 & 12,9 \\
\hline \multirow{3}{*}{ Direito } & ${ }^{134} \mathrm{Cs}$ & 0,0041 & 279 & 1652 & 2834 & 1,4 & 2,3 \\
\hline & ${ }^{137} \mathrm{Cs}$ & 0,0046 & 301 & 1773 & 3041 & 1,6 & 2,7 \\
\hline & ${ }^{106} \mathrm{Ru}$ & 0,0052 & 417 & 2465 & 4260 & 6,4 & 11,1 \\
\hline \multirow{3}{*}{ Esquerdo } & ${ }^{134} \mathrm{Cs}$ & 0,0029 & 397 & 2347 & 4027 & 1,9 & 3,3 \\
\hline & ${ }^{137} \mathrm{Cs}$ & 0,0031 & 440 & 2586 & 4437 & 2,3 & 3,9 \\
\hline & ${ }^{106} \mathrm{Ru}$ & 0,0034 & 632 & 3737 & 6458 & 9,8 & 16,9 \\
\hline
\end{tabular}


Tabela 5. Sensibilidade da técnica para monitoração in vivo de ${ }^{134} \mathrm{Cs},{ }^{137} \mathrm{Cs}$ e ${ }^{106} \mathrm{Ru}$ nos pulmões considerando incorporação de partículas tipo M e AMAD 1 micrômetro, calibração realizada na UCCI com o detetor NaI(TI) 3x3 colimado (Sistema \#2), posicionado na marcação pontual no centro, no pulmão direito e esquerdo do simulador.

\begin{tabular}{|c|c|c|c|c|c|c|c|}
\hline Geometria & Radionuclídeo & $\begin{array}{c}\text { Eff } \\
\text { (cps/dps) }\end{array}$ & $\begin{array}{c}\operatorname{AMD}(\mathbf{B q}) \\
(\mathrm{T}=300 \mathrm{~s})\end{array}$ & $\begin{array}{l}\text { IMD } \\
(\mathrm{Bq}) \\
1 \text { dia }\end{array}$ & $\begin{array}{c}\text { IMD } \\
\text { (Bq) } \\
7 \text { dias }\end{array}$ & $\begin{array}{c}\text { DEMD } \\
1 \text { dia } \\
\left(\mathbf{x 1 0 ^ { - 2 }}\right) \\
(\mathrm{mSv})\end{array}$ & $\begin{array}{r}\text { DEMD } \\
7 \text { dias } \\
\left(\times 10^{-2}\right) \\
(\mathrm{mSv})\end{array}$ \\
\hline \multirow{3}{*}{ Central } & ${ }^{134} \mathrm{Cs}$ & 0,0011 & 102 & 604,2 & 1036,6 & 0,50 & 0,85 \\
\hline & ${ }^{137} \mathrm{Cs}$ & 0,0013 & 96 & 562,5 & 964,9 & 0,50 & 0,85 \\
\hline & ${ }^{106} \mathrm{Ru}$ & 0,0014 & 112 & 660,3 & 1140,9 & 1,72 & 2,98 \\
\hline \multirow{3}{*}{ Direito } & ${ }^{134} \mathrm{Cs}$ & 0,0026 & 44 & 257,9 & 442,4 & 0,21 & 0,36 \\
\hline & ${ }^{137} \mathrm{Cs}$ & 0,0026 & 49 & 287,1 & 492,6 & 0,25 & 0,43 \\
\hline & ${ }^{106} \mathrm{Ru}$ & 0,0027 & 56 & 329,2 & 568,8 & 0,86 & 1,48 \\
\hline \multirow{3}{*}{ Esquerdo } & ${ }^{134} \mathrm{Cs}$ & 0,0010 & 101 & 600,3 & 1030,0 & 0,50 & 0,85 \\
\hline & ${ }^{137} \mathrm{Cs}$ & 0,0011 & 117 & 690,3 & 1184,2 & 0,61 & 1,05 \\
\hline & ${ }^{106} \mathrm{Ru}$ & 0,0013 & 111 & 659,5 & 1139,6 & 0,17 & 2,97 \\
\hline
\end{tabular}

Observa-se na tabela 5 que, para o Sistema \#2, apesar da eficiência de contagem ser menor do que o obtido no sistema \# 1, pelo fato do detetor $\mathrm{NaI}(\mathrm{Tl}) 3 \times 3$ estar colimado, os valores de AMD são menores quando comparados ao Sistema \#1. Isto se explica pela característica de constituição da sala de monitoração, que possui uma construção que apresenta maior efeito de blindagem às radiações de fundo naturais.

Para monitorações realizadas 1 ou 7 dias após a incorporação, a geometria de pulmão direito apresentou maior sensibilidade para o sistema de deteção $\mathrm{NaI}(\mathrm{Tl}) 3 \times 3$ na UCCI pelos mesmos motivos apresentados anteriormente para o Sistema \#1. 
Tabela 6. Sensibilidade da técnica para monitoração in vivo de ${ }^{134} \mathrm{Cs},{ }^{137} \mathrm{Cs}$ e ${ }^{106} \mathrm{Ru}$ nos pulmões considerando incorporação de partículas tipo M e AMAD 1 micrômetro, calibração realizada na UCCI com o detetor NaI(TI) 8x4 (Sistema \#3), posicionado na marcação pontual no centro, no pulmão direito e esquerdo do simulador.

\begin{tabular}{|c|c|c|c|c|c|c|c|}
\hline Geometria & Radionuclídeo & $\begin{array}{c}\text { Eff } \\
\text { (cps/dps) }\end{array}$ & $\begin{array}{l}\text { AMD Bq } \\
(T=300 s)\end{array}$ & $\begin{array}{l}\text { IMD } \\
(\mathrm{Bq}) \\
1 \text { dia }\end{array}$ & $\begin{array}{l}\text { IMD } \\
(\mathrm{Bq}) \\
7 \text { dias }\end{array}$ & $\begin{array}{c}\text { DEMD } \\
1 \text { dia } \\
\left(\mathrm{x10}^{-2}\right) \\
(\mathrm{mSv})\end{array}$ & $\begin{array}{c}\text { DEMD } \\
7 \text { dias } \\
\left(\times 10^{-2}\right) \\
(\mathrm{mSv})\end{array}$ \\
\hline \multirow{3}{*}{ Central } & ${ }^{134} \mathrm{Cs}$ & 0,0169 & 22 & 128,1 & 219,7 & 0,11 & 0,18 \\
\hline & ${ }^{137} \mathrm{Cs}$ & 0,0180 & 23 & 135,3 & 232,1 & 0,12 & 0,21 \\
\hline & ${ }^{106} \mathrm{Ru}$ & 0,0194 & 24 & 142,2 & 245,6 & 0,37 & 0,64 \\
\hline \multirow{3}{*}{ Direito } & ${ }^{134} \mathrm{Cs}$ & 0,0159 & 22 & 132,5 & 227,3 & 0,11 & 0,19 \\
\hline & ${ }^{137} \mathrm{Cs}$ & 0,0170 & 24 & 143,8 & 246,6 & 0,13 & 0,22 \\
\hline & ${ }^{106} \mathrm{Ru}$ & 0,0183 & 25 & 150,9 & 260,8 & 0,40 & 0,68 \\
\hline \multirow{3}{*}{ Esquerdo } & ${ }^{134} \mathrm{Cs}$ & 0,0129 & 28 & 167,2 & 286,8 & 0,14 & 0,24 \\
\hline & ${ }^{137} \mathrm{Cs}$ & 0,0140 & 29 & 172,4 & 295,8 & 0,15 & 0,26 \\
\hline & ${ }^{106} \mathrm{Ru}$ & 0,0153 & 31 & 183,9 & 317,8 & 0,49 & 0,83 \\
\hline
\end{tabular}

No sistema \#3, a sensibilidade na geometria Central é a mais alta, embora muito próxima dos valores obtidos na geometria Direita, e ligeiramente superior à posição esquerda, para os três radionuclídeos avaliados.

De acordo com as tabelas apresentadas, os sistemas de deteção instalados no interior da UCCI são mais sensíveis que o instalado na saleta de alta atividade, como por exemplo, para a detecção de ${ }^{106} \mathrm{Ru}$ na geometria central após 7 dias de incorporação.

Em termos de AMD e DEMD, o Sistema \#3, com detetor de $\mathrm{NaI}(\mathrm{Tl})$ 8x4 é aproximadamente 5 vezes mais sensível que o Sistema \#2, com detetor de $\mathrm{NaI}(\mathrm{Tl}) 3 \times 3$ colimado, e é aproximadamente 20 vezes mais sensível quando comparado com Sistema \#1. Porém este último, com o detetor $\mathrm{NaI}(\mathrm{Tl}) 3 \times 3$ na saleta, pode atingir valores de dose similares aos demais sistemas, quando 
aumentado o tempo de contagem e, consequentemente, havendo o aumento da sensibilidade da técnica.

É importante ressaltar, contudo, que todas as técnicas utilizadas apresentam sensibilidade suficiente para detetar atividades de ${ }^{134} \mathrm{Cs},{ }^{137} \mathrm{Cs}$ e ${ }^{106} \mathrm{Ru}$ que resultem em DEMDs inferiores a $1 \mathrm{mSv}$, de acordo com o cenário de exposição adotado neste trabalho.

A tabela 7 apresenta os valores de contagens líquidas obtidas a partir das medições do radionuclídeo ${ }^{60} \mathrm{Co}$, em duas regiões de energia de emissão gama, quando da realização da validação das geometrias de monitoração em estudo. Foi utilizada a geometria de posicionamento central do detetor para esta verificação e, com base nos resultados obtidos, foi considerada validada a técnica para as demais geometrias. A tabela 8 mostra as eficiências correspondentes para cada sistema de detecção de acordo com suas curvas de calibração.

Tabela 7: Contagens líquidas para o primeiro e segundo pico do ${ }^{60} \mathrm{Co}$.

\begin{tabular}{ccc}
\hline & \multicolumn{2}{c}{ Contagem líquida } \\
\cline { 2 - 3 } Sistema de deteção & RDI 1 & RDI 2 \\
& $\mathbf{1 1 7 3 ~ k e V}$ & 1332 keV \\
\hline 1 - Saleta NaI 3x3 & 14233 & 12033 \\
2 - UCCI NaI 3x3 & 5246 & 4855 \\
3 - UCCI NaI 8x4 & 91539 & 84035 \\
\hline
\end{tabular}

Tabela 8: Eficiências referentes às duas Regiões de Interesse do ${ }^{60} \mathrm{Co}$ correspondentes aos foto picos de 1173 e 1332 keV, para os três sistemas de detecção na posição central.

\begin{tabular}{lll}
\hline \multirow{2}{*}{ Sistema de deteção } & \multicolumn{2}{c}{ Eficiência (cps/Bq) } \\
\cline { 2 - 3 } & RDI 1 & RDI 2 \\
\hline $\mathrm{NaI}(\mathrm{Tl}) 8 \times 4$ / CCI & 0,0140 & 0,0129 \\
$\mathrm{NaI}(\mathrm{Tl}) 3 \times 3$ (col) / CCI & 0,0009 & 0,0008 \\
$\mathrm{NaI}(\mathrm{Tl}) 3 \times 3$ / Saleta & 0,0026 & 0,0023 \\
\hline
\end{tabular}

A sensibilidade do $\mathrm{NaI}(\mathrm{Tl}) 8 \mathrm{x} 4$ é superior aos demais pois o volume deste cristal é maior. Logo em seguida, o detetor que apresenta maior sensibilidade é o $\mathrm{NaI}(\mathrm{Tl}) 3 \times 3$ instalado na saleta de alta atividade. Como discutido anteriormente, a ausência do colimador permite que um maior número de fótons interaja com o cristal em relação ao $\mathrm{NaI}(\mathrm{Tl}) 3 \times 3$ colimado. 
A tabela 9 apresenta as atividades calculadas, utilizando-se, separadamente os dois picos de ${ }^{60} \mathrm{Co}$, bem como as médias das atividades calculadas por cada sistema de detecção e a razão entre as atividades calculadas por meio da técnica aplicada e a atividade de referência do simulador padrão de pulmão contendo ${ }^{60} \mathrm{Co}$.

Tabela 9: Atividades calculadas com base nos picos 1 e 2 do ${ }^{60} \mathrm{Co}$, média das atividades calculadas por cada sistema de deteç̧ão e razão entre atividade calculada e atividade real presente no simulador padrão de pulmão.

\begin{tabular}{lcccc}
\hline \multirow{2}{*}{ Sistema de deteção } & \multicolumn{2}{c}{ Atividade calculada } & \multirow{2}{*}{ Média das Atividades } & $\mathrm{R}$ \\
\cline { 2 - 4 } & ${ }^{60} \mathrm{Co}(1)$ & ${ }^{60} \mathrm{Co}(2)$ & & \\
\hline CCI NaI 8x4 & 21853 & 21722 & 21788 & 0,97 \\
CCI NaI 3x3 (col) & 19874 & 20558 & 20216 & 0,90 \\
Saleta NaI 3x3 & 18099 & 17377 & 17738 & 0,79 \\
\hline
\end{tabular}

A razão (R), calculada para os três sistemas de detecção avaliados, apresenta valores na faixa 0,79 a 0,97 . O sistema de deteção \#1, instalado na saleta de alta atividade, foi o que apresentou maior discrepância em relação à atividade de referência, com diferença superior a 20\%. Entretanto, considerando-se a variedade de fatores que interferem na exatidão deste tipo de técnica [7] este valores de " $\mathrm{R}$ " podem ser considerados admissíveis para uma técnica de monitoração in vivo. Devese destacar também que tais discrepâncias seriam aceitáveis em exercícios de intercomparação, estando dentro dos limites de tolerância internacionais [8].

Com base nos resultados obtidos neste trabalho, comparativamente, a sala blindada do Contador de Corpo Inteiro é o local mais apropriado para medição da atividade corporal. Entretanto, em uma situação real de emergência, o sistema portátil (\#1) seria mais adequado para ser utilizado em uma primeira avaliação da severidade do acidente em termos de incorporação de radionuclídeos.

Com relação às incertezas associadas à técnica, é importante ressaltar que trata-se de uma metodologia a ser aplicada em situações de emergência. E caso sejam detetadas incorporações relavantes, medições mais detalhadas seriam recomendadas a serem executadas nos indivíduos envolvidos no acidente.

A avaliação de técnicas de monitoração in vivo requer uma série de experimentos específicos complementares a fim de identificar as incertezas do Tipo A e B e seu peso na incerteza combinada 
total do processo de monitoração. O Guia IDEAS [7], editado pela Comunidade Europeia, fornece uma estimativa dos fatores que contribuem para a incerteza associada às técnica de monitoração in vivo. O chamado scatering factor total, no caso de radionuclídeos que emitem fótons da alta energia, é estimado da ordem de 1,2 dependendo do sistema de deteção e da geometria de medição. A tabela 10 apresenta uma estimativa do fator de disperção total da presente técnica, por meio de uma composição dos valores de $\mathrm{sf}_{\mathrm{i}}$ sugeridos pelo Guia IDEAS e dos parâmetros específicos, Pico Soma, Atividade do padrão e Meia-vida física.

Tabela 10. Fatores de dispersão $\left(\mathrm{sf}_{\mathrm{i}}\right)$ típicos da monitoração in vivo de radionuclídeos emissores de fótons com energia > $100 \mathrm{keV}$.

\begin{tabular}{|l|c|}
\hline Componentes de incerteza (tipo) & $\mathrm{sf}_{\mathrm{i}}$ \\
\hline Estatística das contagens (A) & 1,07 \\
\hline Posicionamento do detetor (B) & 1,05 \\
\hline Sinal do background (B) & 1,05 \\
\hline Espessura da camada torácica (B) & 1,12 \\
\hline Distribuição da atividade (B) & 1,05 \\
\hline Dimensão do corpo (B) & 1,07 \\
\hline Pico soma (B) & 1,05 \\
\hline Atividade do padrão (B) & 1,01 \\
\hline Meia-vida (B) & 1,001 \\
\hline Fator de dispersão total (sf) & 1,17 \\
\hline
\end{tabular}

\section{CONCLUSÕES}

A geometria mais apropriada para a monitoração em situações de emergência dependerá das propriedades biocinéticas do radionuclídeo de interesse. No caso de exposição via inalação, tema deste trabalho, a geometria recomendada seria o posicionamento do detetor direcionado ao centro do tórax, quando há contribuição de ambos os pulmões na taxa de contagem registrada. As geometrias Direita e Esquerda são mais susceptíveis a erros relacionados à diferença entre o 
formato do corpo dos indivíduos medidos e o simulador utilizado para a calibração. Como exemplo pode-se citar a monitoração de pessoas obesas e mulheres. Ocorre que quanto maior o volume das mamas, maior será a atenuação da radiação e menos interações ocorrerão no volume sensível do detetor, diminuindo a eficiência das deteção. Deve-se ressaltar que o simulador de tórax utilizado na calibração não prevê a diferença de atenuação devido à presença das mamas. Além disso, destaca-se o fato de que a diferença de valores apresentados nos resultados para as diferentes geometrias são irrelevantes do ponto de vista da monitoração interna. Assim, por uma questão de praticidade, a geometria central é a mais adequada para este tipo de monitoração em situações de emergência.

Baseado na avaliação da sensibilidade das técnicas estudadas, expressas pelas DEMDs obtidas através das calibrações realizadas para os diferentes sistemas de detecção, conclui-se que, para uma monitoração realizada até 7 dias após a incorporação, o sistema de deteção \#3 ( $\mathrm{NaI}(\mathrm{Tl})$ 8x4 instalado no CCI) é o mais sensível para monitoração dos três radionuclídeos, ${ }^{134} \mathrm{Cs},{ }^{137} \mathrm{Cs}$ e ${ }^{106} \mathrm{Ru}$. Este resultado já era esperado e está diretamente relacionado às dimensões do cristal de $\mathrm{NaI} 8 \times 4 \mathrm{em}$ relação aos demais detetores avaliados.

A técnica utilizando o $\mathrm{NaI}(\mathrm{Tl}) 3 \times 3$ na saleta de alta atividade foi a que apresentou menor sensibilidade. Isto se deve à maior taxa de contagem da radiação de fundo em relação ao interior da sala blindada da UCCI. Entretanto o seu uso é factível em situações de emergência já que, apesar de apresentar menor sensibilidade, mesmo assim os valores de DEMD situam-se na ordem de milisieverts. Nestas situações, a rapidez na obtenção de resultados é fundamental e sua principal característica é a facilidade de transporte do sistema, peculiaridade que os demais sistemas não possuem. Baseado neste critério esta técnica também pode ser utilizada em situações de emergência, mesmo que não seja tão sensível quanto as demais técnicas.

Com base nos valores estimados de DEMD para os cenários de exposição simulados neste trabalho, conclui-se que as técnicas utilizadas são capazes de detetar níveis de atividades de ${ }^{134} \mathrm{Cs}$, ${ }^{137} \mathrm{Cs}$ e ${ }^{106} \mathrm{Ru}$ nos pulmões, decorrentes de incorporações que resultam em doses efetivas com valores na ordem de $10^{-2} \mathrm{mSv}$, para todos os sistemas propostos neste trabalho. Sendo assim, a metodologia é válida para sua utilização em situações de emergências com incorporação via inalação dos radionuclídeos citados, supondo monitoração realizada até 7 dias após o acidente. 


\section{AGRADECIMENTOS}

À CAPPES, pela bolsa de estudos e à FINEP/MCTIC (Projeto 01.10.0650.01), pelo suporte financeiro necessário à aquisição de simuladores antropomórficos de pulmão utilizados na calibração dos detetores.

\section{REFERÊNCIAS}

[1] IAEA - International Atomic Energy Agency. Methods for Assessing Occupational Radiation Doses due to Intakes of Radionuclides. Safety Reports Series No. 37, 2004

[2] UNSCEAR. Levels and effects of radiation exposure due to the nuclear accident after the 2011 great east-Japan earthquake and tsunami. 2013

[3] BERTELLI, L.; MELO D. R.; LIPSZTEIN J.; CRUZ-SUAREZ R.; “AIDE: Internal Dosimetry Software", Radiation Protection Dosimetry, 130(3): 358-367, 2008

[4] OLIVEIRA, C.A.N.; LOURENÇO, M.C.; DANTAS, B.M.; LUCENA, E.A.; LAURER, G.R., "The IRD/CNEN whole body counter: Background and calibration results", Radiation Protection Dosimetry, 29(3): 203-208, 1989

[5] MELLO, J.Q.; DANTAS, A.L.A.; ALMEIDA, A.P.F.; HUNT, J.G.; DANTAS, B.M., Comparação entre métodos de simulação física e matemática para calibração de detetor de cintilação, visando à determinação da radionuclídeos de alta energia no corpo humano, Brazilian Journal of Radiation Sciences, 3(1): 1-5, 2015

[6] EL-KATHIB, A.M. et al. Full energy peak efficiency of an $\mathrm{NaI}(\mathrm{Tl})$ detector with coincidence summing correction showing the effect of the source-to-detector distance. Chinese journal of Physics, 55: 478-489, 2017

[7] CASTELlANi, C.M, MARSH, J.W., HURTGEN, BLANCHARDON, E., BERARD P., GIUSSANI A., LOPEZ, M.A., 2013. IDEAS Guidelines (Version 2) for the Estimation of Committed Doses from Incorporation Monitoring Data. EURADOS Report 2013-01 Braunschweig.

[8] ISO/IEC 17025:2005. General requirements for the competence of testing and calibration laboratories. 